\title{
Malignant Nerve Sheath Tumor with Secondary Intradural Extramedullary Metastases \\ (Case Report)
}

Authors

\section{Ahmed Hassan Samsudeen, Pravin GU, Parthasarathi A, Javaji Raviprasad}

\begin{abstract}
We present a case of a 65 year old male patient of a malignant nerve sheath tumor with multiple intradural, extra medullary metastases and its preoperative imaging on MRI. Patient presented with complaints of pricking type of pain the cervical and dorsolumbar region since one year which was progressive since the day of onset of the symptoms.
\end{abstract}

\section{Introduction}

Nerve sheath tumors account for 80 to $90 \%$ of all intradural extramedullary neoplasms ${ }^{1}$. Two main types of nerve sheath tumors are found in the spine: schwannoma (also known as neurinoma or neurilemoma) and neurofibroma. Schwannomas are lobulated, grossly encapsulated, well circumscribed round or oval tumors that often show cystic degeneration, hemorrhage and xanthomatous changes. Schwannomas arise eccentrically from their parent nerve; primary malignant peripheral nerve sheath tumors occur but are very rare ${ }^{2}$. Schwannomasal most never become malignant whereas sarcomatous transformation occurs in $4 \%$ to $11 \%$ of patients with neurofibromatosis 3 .

\section{Case Presentation}

A 65 year old male patient presented to outpatient department of orthopedic complaining of a pricking type of pain in the cervical and dorso lumbar region since one year and was progressive since the day of onset of the symptoms.

Physical examination demonstrated loss of patient strength of the right arm with restriction of movements.

The patient was referred to the department of Radio-diagnosis for imaging workup, An MRI Study of the cervical spine was requested, imaging was conducted using 1.5 Tesla Siemens MR Machine.

Axial GRE, sagittalT1W and T2W sequences were taken at the level of the cervical spine with screening of the brain and whole spine. MRI of the cervical spine revealed two intradural extramedullary lesions, the larger lesion was well defined which was present at the level of the foramen magnum, closely adherent to the brain stem. The lesion was indenting cerebellar tonsils with outaltered signal intensities in the basion, ophisthion and the posterior elements. Another lesion was noticed at the level of C6 \& C7 vertebral bodies, the lesion was well defined and 
was displacing the spinal cord. The exiting and traversing nerve roots at the level of C6 - C7 IV disc could not be delineated from the lesion. The facet joints, pre and paravertebral soft tissues and ligaments were normal

Contrast study was done by administering $10 \mathrm{ml}$ of gadobenatedimeglumine intravenously with showed intenseheterogeneous contrast enhancement of both lesions. .

Further investigation prompted the screening of the entire brain which showed no intraaxial/ extraaxiallesion or abnormality.

Localizer of lumbar spine showed lesion at the level of L3 \& L4 vertebral bodies in left paraspinal region. Further imaging in axial and sagittal plans were performed which showed welldefined heterointense lesion, showing heterogeneous contrast enhancement with the displacement of the psoas muscle. The lesion was placed well away from the inferior pole of the right kidney. The lesion was not causing any mass effect over the neural foramen, the lesion could not be delineated from the nerve.

After the initial work up of the patient the provisional diagnosis that was considered before the initiation of the diagnostic studies were degenerative changes of the spine with intervertebral disc prolapse.

In this patient, even though multiple lesions were seen in the dural sac before the imaging of the paraspinal lesion which was an incidental finding the patient had increase signal intensities of the vertebral body in the cervical and dorsal vertebrae with normal signal intensities of the posterior elements and in view of no lesions noted in the brain and follow up abdominal ultrasound and chest radiograph was insignificant. The possibility of the lesion to be a metastasis was contradicting the imaging because the primary source could not be found.

Different diagnostic hypotheses was formulated from schwannoma, neurofibroma, multiple metastasis, meningioma, neurofibromatosis -II and malignant nerve sheath tumor.

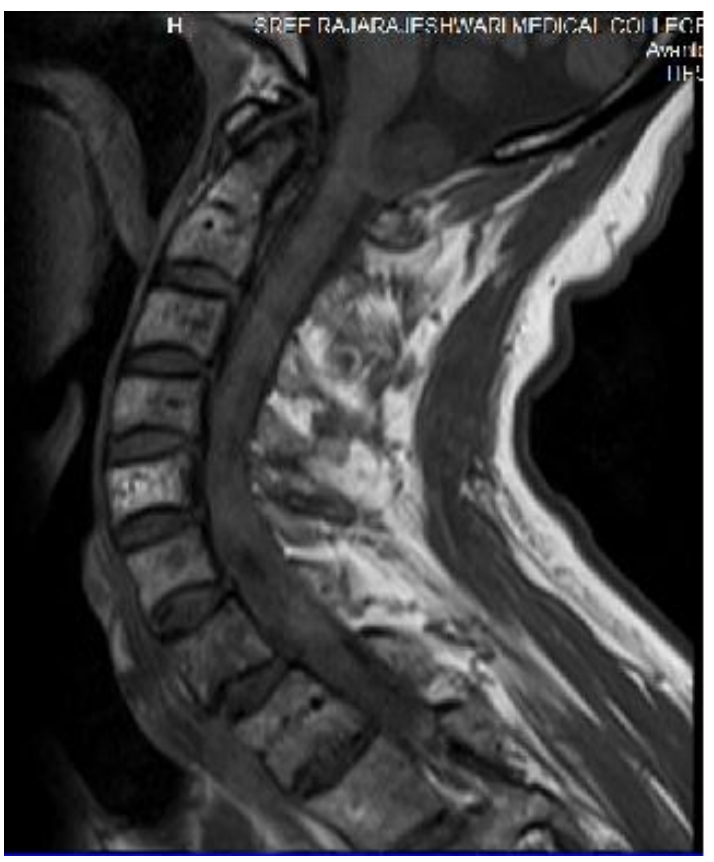

Fig 1: T1w Sagital Image Showing Two Intradural Extramedullary Lesions with Displacement Of The Adjacent Cord With High Signal Intensity Of The C4 Vertebral Body.

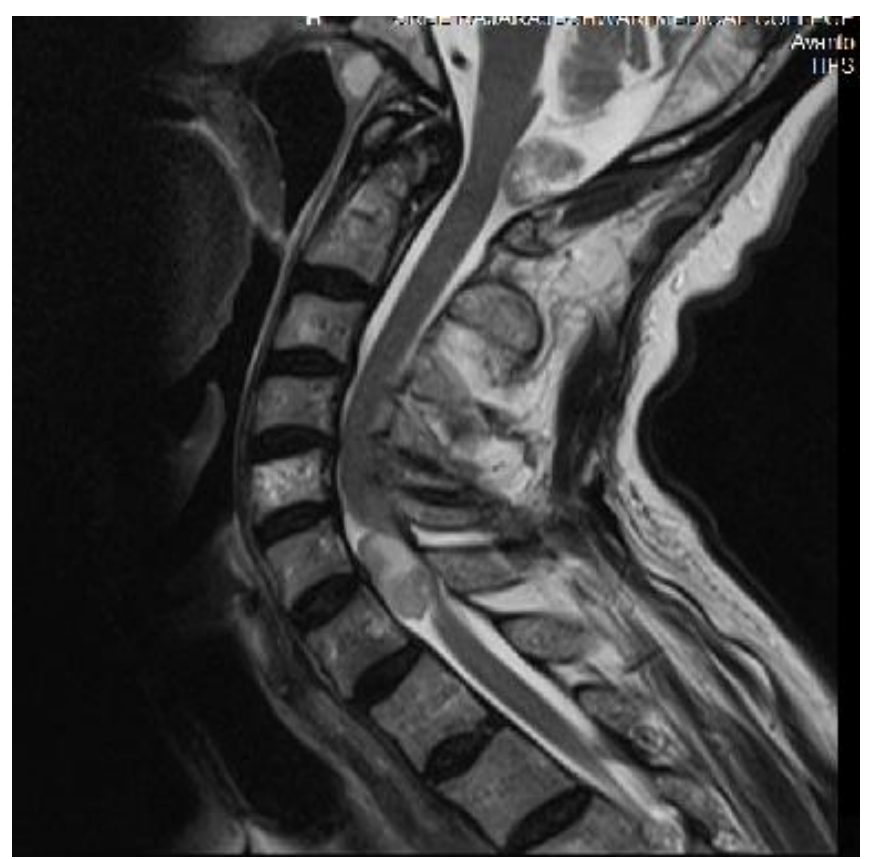

Fig 2: T2w Sagital Image Showing Two Intradural Extramedullary Lesions Of High Signal Intensity. 


\section{JMSCR Vol||04||Issue||04||Page 10039-10043||April}

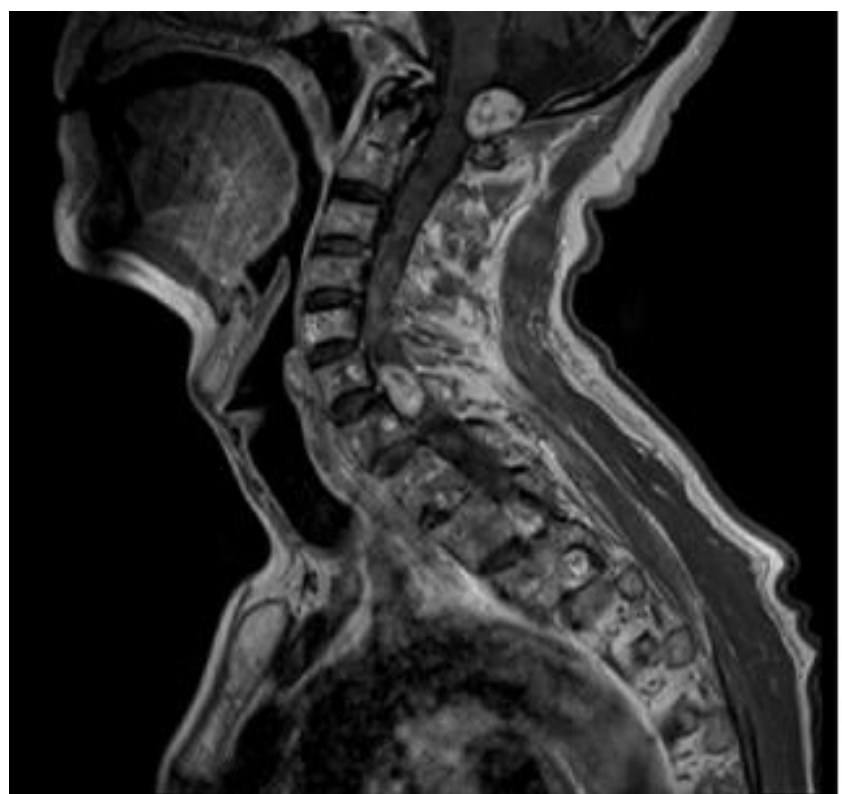

Fig 3: T1w Sagital Post Contrast Image Showing Enhancement Of The Lesion With Areas Of Low Signal Intensities Within The Lesions.

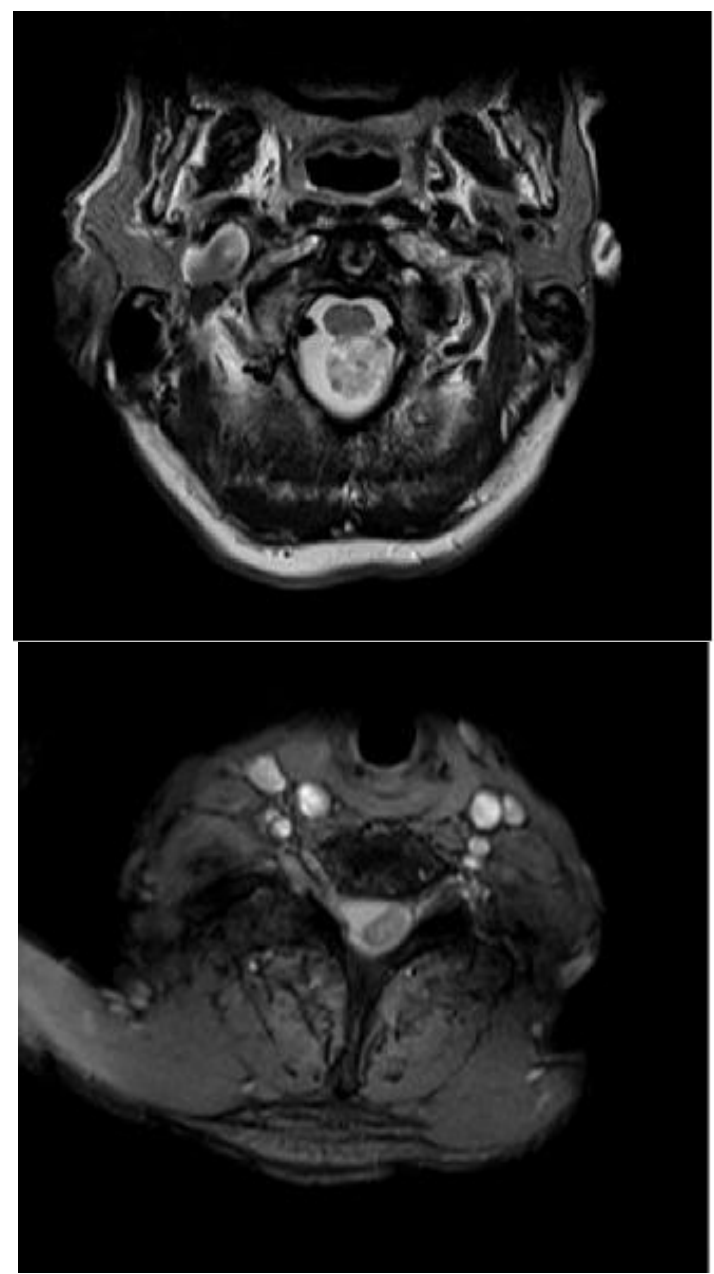

Fig 4 : Axial Images Showing The Intradural Extramedullary Lesion With Displacement Of The Cord.

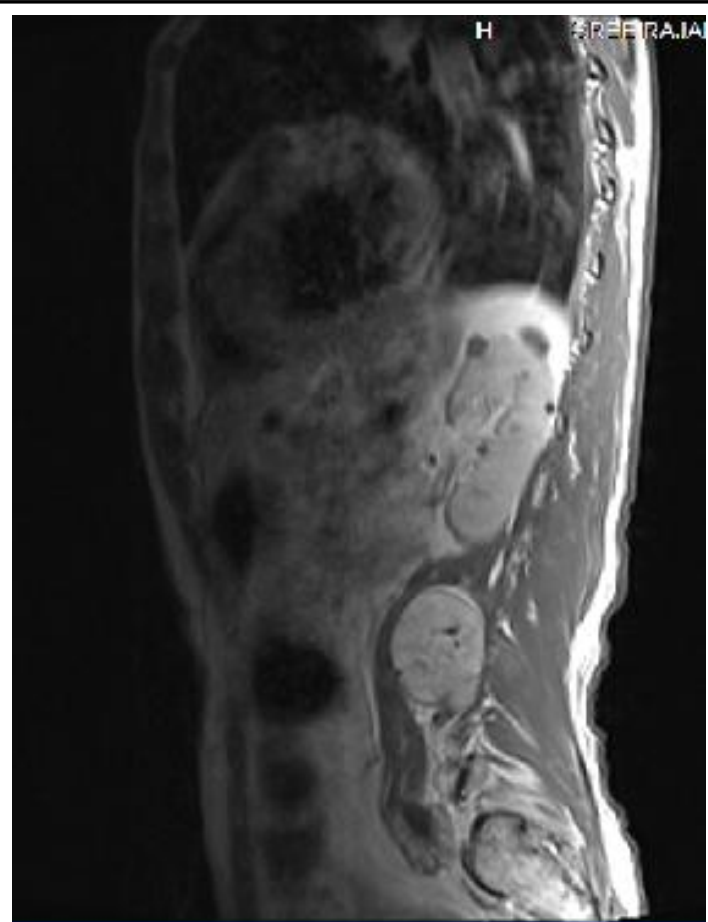

Fig 5: T1w Sagital Image Showing A Hyperintense Lesion Arising From The Lumbar Nervous Plexus With Displacement of The Psoas Muslce.

\section{Discussion}

Schwannomas are often seen as solitary lesion and rarely are multiple, they are seen in the lower extremity, followed by the torso and retroperitoneum and rarely undergo malignant transformation. Radiologically the mass is always placed eccentric relative to the nerve and is inseparable, schwanomas are commonly capsulated tumors and intratumoral cysts with the presence of a target sign on post contrast is seen Primary metastasis or drop metastasis can occur from a primary tentorial lesion such as cerebral glioblastoma, anaplastic astrocytoma, ependymoma, posterior fossa medullablastoma, pineal tumors and choroid plexus tumors serve as the primary lesion. Clinically a patient with drop metastasis can present with the brown sequard syndrome which is a result of the hemicord damage.

Other non CNS causes of spinal metastasis is solid tumors arising from the lung or breast, hematopoietic pathologies such as leukemia, lymphoma and melanoma. 
Meningioma's are benign tumors and is usually seen the middle aged female population, they present clinically as motor or sensory deficits and pain is presented as local, radicular or funicular type of pain. Spinal meningioma's are slow growing neoplasms, which are usually solitary. Multiple meningiomas are seen in neurocutaneous syndrome like neurofibromatosis type II.

Neurofibromas are typically associated as solitary lesions when they occur as multiple lesions, neurofibromatosis type $\mathrm{I}$ is a strongly associated with it.NF1 is the most common of the phakomatoses (neurocutaneous syndromes) and occurs in one of every 2,000 live births. NF1 is inherited as an autosomal dominant disorder; however, up to $50 \%$ of cases occur sporadically due to spontaneous mutation. Although many affected patients present in childhood with classic clinical findings, up to $10 \%$ of patients present later in life with formefruste ${ }^{8}$ or atypical manifestations They are most often seen in the head and neck, lower extremity, torso, the mass is placed relatively central to the nerve and is intimately related to the affected nerve. Cysts are rarely seen in neurofibromas and they are well circumscribed lesions showing the peripheral contrast enhancement.

Malignant nerve sheath tumors are seen in the major nerve trunks as seen in this patient and the mass is central and relative to the nerve and infiltrates the nerve, these lesions do not have a capsule and they are well circumscribed lesions rather than with irregular borders commonly seen in the age group of 25 to 65 years of age. They account for $3-10 \%$ of all soft tissue sarcomas ${ }^{4,5}$. Malignant nerve sheath tumors have a tendency to recur locally and to metastasize and are known to be highly malignant ${ }^{6}$.Malignant neural neoplasms arise either from neurofibromas on de novo from nerve sheaths. Origin from a benign schwannoma is exceedingly rare ${ }^{5,7}$. The varied terminology applied to malignant neural neoplasms, including malignant schwannoma, malignant neurilemoma, nerve-sheath fibrosarcoma, neurogenic sarcoma, and neurofibrosarcoma, reflects the controversy surrounding their histogenesis. Since the precise cell of origin of these neoplasms is unknown, they are best called malignant peripheral nerve-sheath neo- plasms $^{7}$

The patient did not present with any cutaneous manifestation such as café au lait spots. MRI brain did not reveal any mass of cranial nerve. Thevestibulocochlear and trigerminal nerve appeared normal and patient did not give any familial history relating to neurofibromatosis type II.

The larger intradural lesion which was noted in the foramen magnum was taken into consideration and posterior extramedullaryintradural masses including ependymoma, subependymoma and medulloblastomas was ruled out, where these lesions occurring as multiple lesions was unlikely. Non glial causes of metastasis was also ruled out by taking a chest radiograph and abdominal ultrasound which showed no significant abnormality.

The lesion was concluded as a malignant nerve sheath tumor that was arising from nerve trunk with multiple secondaries seen as intraduralextramedullary lesions

\section{Reference}

1. Li Mh, Holtas , Larsson E - M: MR imaging of intradural extramedullary tumor, acta radiology 33.207-212,1992

2. Anne G Osborn, Diagnostic radiology third reprint pg895

3. Barboriak Dp,Rivitz SM, Chew FS: sacral neurofibroma, AJR 159:600,1992

4. Grobmyer SR, Reith JD, Shahlaee A, Bush $\mathrm{CH}$, Hochwald SN. Malignant peripheral nerve sheath tumor: molecular pathogenesis and current management considerations. J SurgOncol 2008; 97:340-349

5. Enzinger FM, Weiss SW. Malignant tumours of peripheral nerves. In: Enzinger FM, Weiss SW, eds. Soft tissue tumors, vol. 31. St. Louis, MO: Mosby Company, 2001:1209-1263 
6. Junjiwasa, Yoshiro wada, MRI features in the differentiation of malignant peripheral nerve sheath tumors and neurofibromas AJR 190, Page 204

7. Woodruff JM, Horten BC, Erlandson RA. Pathology of peripheral nerves and paragangiiomas. in: silverberg SG, ed. Principles and practice of AJR:149, November 1987 surgicalpathology, vol. 2. New York: Wiley, 1983:1503-1520

8. Biondetti PR, Vigo M, Flore D, et al. $C T$ appearance of generalized von Recklinghausen neurofibromatosis. $J$ Comput Assist Tomogr 1983; 7:866-869 